\title{
Generalized peeling skin syndrome
}

INSERM

\section{Source}

INSERM. (1999). Orphanet: an online rare disease and orphan drug data base.

Generalized peeling skin syndrome. ORPHA:263543

Generalized peeling skin syndrome (PSS) is a form of PSS (see this term) presenting with a generalized distribution. It comprises two sub-types: the non-inflammatory (PSS type A) and the inflammatory (PSS type B) form (see these terms). PSS type A is characterized by generalized white scaling with superficial peeling of the skin, while PSS type B is characterized by superficial patchy peeling of the entire skin with underlying erythroderma, associated with pruritus, and atopy. 